\title{
¿Por qué los programas de transferencias condicionadas \\ no consiguen llegar a las personas más pobres? El caso de las zonas urbanas en México
}

\author{
Pierre Levasseur
}

\section{Resumen}

Dada la limitada capacidad de financiación de los países en desarrollo, los programas de transferencias condicionadas (PTC) constituyen un modo asequible de proporcionar una red de seguridad social para hogares vulnerables. Sin embargo, su condicionalidad puede limitar la participación e incrementar su abandono, en especial cuando las imposiciones asociadas al cumplimiento son elevadas y los incentivos en efectivo relativamente bajos. Este análisis empírico determina cómo los montos de las transferencias en efectivo afectan la probabilidad de que los hogares permanezcan en un programa o lo abandonen, estudiando el caso de México: un país en desarrollo que ha expandido de manera progresiva su PTC desde las zonas rurales a las urbanas. Mediante encuestas de hogares longitudinales, este estudio concluye que los hogares más pobres tienen más probabilidades de abandonar el programa. Es más, el monto de las transferencias monetarias incrementa esta probabilidad para los participantes más pobres en comparación con los más ricos. Se concluye que el programa no consigue retener a los hogares más pobres porque los incentivos en efectivo son demasiado bajos para los entornos urbanos.

\section{Palabras clave}

Ingresos, hogares, mitigación de la pobreza, desarrollo económico, zonas rurales, zonas urbanas, programas de acción, evaluación de programas, estudios de casos, México

\section{Clasificación JEL}

O15, I32, H23

\section{Autor}

Pierre Levasseur es Investigador Asociado en la Unidad de Ciencias para la Acción y el Desarrollo - Actividades, Productos, Territorios (SAD-APT) del Instituto Nacional de Investigación para la Agricultura, la Alimentación y el Medio Ambiente de Francia (INRAE). Correo electrónico: pierre.levasseur@inrae.fr. 


\section{Introducción}

En los países en desarrollo, que suelen estar sobreendeudados, las restricciones fiscales y presupuestarias limitan la implementación de sistemas de protección social. Muchos países emergentes, como México, han optado por una solución alternativa: los programas de transferencias condicionadas (PTC). Dado que estos programas están dirigidos únicamente a los hogares más pobres, su costo es relativamente bajo. La premisa es sencilla: los participantes aceptan respetar determinadas condiciones a cambio de un subsidio en efectivo (o prestaciones no monetarias), con el objetivo general de incrementar la demanda de servicios públicos (por ejemplo, en escuelas y centros sanitarios). Se dice que las transferencias monetarias son "condicionadas" porque los participantes que no cumplen las condiciones quedan excluidos del programa y dejan de recibir pagos. Los incentivos en efectivo tienen dos funciones principales: i) fomentar la participación de los hogares admisibles en el programa, y ii) compensar las imposiciones derivadas de la utilización de servicios públicos, tales como sus costos directos (por ejemplo, tasas de matriculación, uniformes, equipamiento, consultas médicas, transporte, medicamentos, inyecciones) e indirectos (como el costo de oportunidad o la inversión de tiempo). Las personas que viven en la pobreza son especialmente vulnerables a este tipo de imposiciones. Evidentemente, existen más factores que limitan la demanda de servicios públicos por parte de las poblaciones en situación de pobreza, como, por ejemplo, la incapacidad para priorizar la salud y la educación. Teóricamente, esto podría atribuirse a una falta de conocimientos en materia nutricional y educativa, así como a la incoherencia temporal (preferencia por la gratificación inmediata frente a una inversión futura incierta en capital humano (Banerjee y Duflo, 2011).

EI PTC mexicano, llamado inicialmente Progresa, después Oportunidades y conocido ahora como Prospera, es uno de los más ambiciosos y de mayor éxito a nivel mundial (Fiszbein y otros, 2009). En un primer momento el propósito del programa era erradicar el hambre y la pobreza extrema a través de acciones en el ámbito de la educación, las deficiencias nutricionales y las enfermedades infecciosas. Desde su puesta en marcha en 1997 como parte de un ensayo aleatorio controlado en unas pocas zonas rurales marginadas, el programa ha demostrado tener un impacto positivo en los indicadores de capital humano. A la vista de sus resultados concluyentes, el Gobierno decidió extender el proyecto a todas las áreas rurales marginadas del país entre 1998 y 2000, y después a pequeñas zonas urbanas en 2001 y a las principales ciudades de México en 2002. Desde 2007, los hogares pobres de todas las comunidades marginadas del país - tanto en zonas rurales como urbanas - pueden participar en el programa.

Pese a su éxito en zonas rurales de México en lo que respecta a la educación, la salud y la nutrición, sobre todo de niños y niñas (Hoddinott y Bassett, 2008), la literatura pone de manifiesto que la ampliación del programa a zonas urbanas desde 2001 se ha visto sometida a diversas limitaciones. La participación entre los hogares urbanos admisibles en el programa (o índice de utilización) ha sido reducida (en torno al 50\%). Según Angelucci, Attanasio y Di Maro (2012) y Behrman y otros (2012), existen varias razones que explican el elevado nivel de errores de exclusión en las ciudades. En primer lugar, el proceso de autoselección a cargo de los participantes se ha traducido en una serie de exclusiones involuntarias. Del 40\% de hogares admisibles que no solicitaron participar en el programa, un tercio de ellos declararon desconocer su existencia. Esto significa que casi un 30\% de los hogares urbanos admisibles decidieron voluntariamente no inscribirse (es decir, se autoexcluyeron). Además, el índice anual de abandono del programa, motivado fundamentalmente por el incumplimiento de las condiciones, supera el 7\% en las zonas urbanas, frente al 3\% en las zonas rurales. Llama la atención que, en contra de la teoría de la autofocalización, los hogares urbanos más pobres presentan más probabilidades de abandonar el programa (González-Flores, Heracleous y Winters, 2012). En línea con la teoría sobre la utilización, los hogares admisibles parecen adoptar una decisión racional entre las prestaciones del programa y los costos que implica adherirse al mismo. Así pues, además de los criterios requeridos 
para participar en el programa y el desconocimiento de su existencia, el otro factor que podría explicar el bajo índice de utilización y la escasa participación entre los hogares urbanos admisibles más pobres (así como la dificultad para que permanezcan en el programa) es el desequilibrio entre los incentivos en efectivo y el costo de la vida en las ciudades; el valor de las transferencias monetarias es el mismo en zonas rurales y en zonas urbanas (Angelucci y Attanasio, 2009), pero el costo de la vida es muy diferente. De este modo, los incentivos en efectivo de los programas parecen ser demasiado bajos como para fomentar la participación de una parte significativa de los hogares urbanos admisibles y para compensar las imposiciones derivadas de su condicionalidad (costos directos e indirectos, preferencia por una gratificación inmediata y otros). Por extraño que parezca, no existen estudios empíricos acerca del modo en que el monto de las transferencias monetarias influye en los índices de utilización y abandono de los programas. Sea como fuere, el valor de los incentivos en efectivo es una variable ajustable que puede mejorar ambos índices de manera más que proporcional (Attanasio, Meghir y Schady, 2010).

Este estudio parte de un diseño cuantitativo basado en datos de panel procedentes de la Encuesta Nacional sobre Niveles de Vida de los Hogares (ENNVIH) y pretende comprender mejor los factores que determinan el bajo índice de utilización que caracteriza a la versión urbana del PTC mexicano. Más concretamente, este es el primer estudio que evalúa la influencia directa de los incentivos en efectivo sobre los riesgos de abandono del programa, y considera para ello un amplio abanico de factores determinantes de la utilización. La identificación de factores determinantes del abandono resultará de utilidad para los administradores de estos programas y les permitirá minimizar los errores de exclusión. En efecto, los errores de exclusión son especialmente elevados en las ciudades: tres cuartas partes de los abandonos del programa se producen como consecuencia de un incumplimiento de las condiciones asociadas a los requisitos sanitarios y nutricionales y de otros requisitos de procedimiento (GonzálezFlores, Heracleous y Winters, 2012).

El resto del artículo se estructura del siguiente modo: la sección II resume el PTC mexicano, la sección III describe la literatura en que se ha analizado el bajo índice de utilización del programa en áreas urbanas, en la sección IV se describen los datos y se establece la estrategia empírica, en la sección V se comentan los resultados y, por último, la sección VI recoge algunas conclusiones.

\section{El programa de transferencias condicionadas (PTC) de México}

\section{Prestaciones}

El programa mexicano ofrece dos tipos de transferencias monetarias: i) ayuda básica para incrementar el consumo de alimentos en el hogar y ii) becas opcionales para fomentar la asistencia escolar. Cada una de ellas está asociada a unas condiciones específicas.

A cambio de la ayuda básica para alimentos (cuyo monto es el mismo para todos los participantes), los miembros que componen el hogar deben visitar el centro médico más cercano cada dos meses para someterse a un chequeo médico. La persona que recibe las transferencias (por lo general, la madre) también debe asistir a sesiones públicas mensuales de capacitación en materia de salud y sobre salud preventiva o nutrición (que reciben el nombre de pláticas). Los centros médicos informan todos los meses a los administradores del programa sobre la asistencia a estas reuniones. Los hogares que incumplan estas condiciones cuatro meses consecutivos (o seis meses no consecutivos en el plazo de un año) pierden automáticamente su derecho a todas los prestaciones del programa.

En lo que respecta a las becas escolares, el monto de la transferencia varía según el número de menores en edad escolar que haya en el hogar, el curso escolar al que asistan, así como su género 
(véase el cuadro A1.1 en el anexo A1). El programa establece un monto máximo de transferencia (Skoufias, 2005), probablemente para evitar posibles incentivos a la fertilidad que resultarían adversos. Para beneficiarse de una beca completa, el estudiante debe matricularse en una escuela aprobada por la Secretaría de Educación Pública (SEP) y asistir al menos al 85\% de las clases al mes. La dirección de la escuela informa a los administradores del programa sobre la matriculación y la asistencia. Si la asistencia es inferior al 85\%, se deduce una cantidad proporcional del pago bimensual. Por ejemplo, si un estudiante se ausentó del 50\% de las clases en los dos meses anteriores, el hogar recibirá un $50 \%$ del monto de la beca.

Para concluir, al contrario de lo que sucede con la transferencia básica para alimentos, las condiciones asociadas a la beca no implican necesariamente exclusiones definitivas, sino reducciones en los pagos (Álvarez, Devoto y Winters, 2008). Esto supone que un hogar puede adherirse solo a una parte del programa, siempre que se satisfagan las condiciones sanitarias y nutricionales. No obstante, en caso de que un hogar incumpla sus obligaciones derivadas del componente sanitario y nutricional, aunque siga enviando a sus hijos a la escuela perderá de manera permanente su derecho a todas las prestaciones del programa.

\section{El proceso de selección de beneficiarios}

La estrategia de identificación de beneficiarios del PTC mexicano incluye un proceso de selección dual. En primer lugar, se realiza una selección geográfica de las comunidades más pobres. Puesto que el nivel de concentración de hogares pobres varía mucho entre las zonas rurales y las urbanas, el proceso de selección geográfica es específico para cada región (Orozco y Hubert, 2005). Los hogares admisibles se encuentran en comunidades rurales y áreas urbanas preseleccionadas. Dicho de otro modo, solo pueden participar hogares pobres que pertenezcan a comunidades pobres.

En comunidades rurales seleccionadas que cumplían los requisitos para participar en el programa se realizaron entrevistas en persona en los hogares de los potenciales beneficiarios. De este modo, se llevó a cabo un censo cuasi exhaustivo de los habitantes de las zonas rurales. Se invitó a participar a los hogares rurales que reunían los criterios de adhesión. Sin embargo, por razones de costo este proceso no se ha podido replicar en las áreas urbanas; en su lugar, se ha establecido un sistema de autoselección. Los hogares de las áreas urbanas admisibles al programa han sido informados de su existencia por medio de mensajes transmitidos por altavoz desde automóviles que circulaban por determinados vecindarios, en la publicidad y por la radio. Los hogares que se consideren admisibles al programa deben acudir a su sucursal temporal más cercana (ubicadas en centros de salud), donde se los entrevistará en el marco de la Encuesta de Características Socioeconómicas de los Hogares Urbanos (ENCASURB). La información se introduce directamente en un sistema informático y se contrasta de manera simultánea con un índice de pobreza multivariante (homogéneo en todo el territorio nacional desde 2001), de modo que se genera un puntaje (véase Skoufias, Davis y De la Vega, 2001). Por último, se efectúan visitas a aquellos hogares que reúnan las condiciones para participar (con un puntaje superior a 0,69) con objeto de comprobar la exactitud de los datos facilitados durante la entrevista. Cada tres años, los administradores del programa efectúan un proceso de recertificación para limitar los errores de inclusión. Durante la recertificación se vuelve a evaluar el puntaje a partir de información actualizada sobre el hogar. Los hogares con un puntaje por encima de 0,383 siguen siendo beneficiarios plenos, los hogares con un puntaje por debajo de 0,077 quedan totalmente excluidos, y los hogares con un puntaje entre 0,077 y 0,383 son transferidos a una versión reducida del programa (denominada Esquema Diferenciado de Apoyos (EDA)) durante otros tres años, antes de quedar excluidos. Los hogares transferidos al EDA siguen recibiendo prestaciones en materia nutricional, el paquete sanitario básico y becas para estudiantes de educación secundaria. 
Este sistema de autoselección se ha traducido en la exclusión de muchos hogares urbanos admisibles. Angelucci, Attanasio y Di Maro (2012) estiman que cerca de un 50\% de los hogares urbanos potencialmente admisibles no se están beneficiando del programa, frente a menos del $10 \%$ en el caso de hogares rurales. Behrman y otros (2012) informan que en torno a un tercio de los hogares urbanos admisibles que no participan no conocían la existencia del programa. Dicho de otro modo, más de dos tercios de los hogares admisibles no participantes decidieron libremente no adherirse a pesar de poder hacerlo. Si bien es probable que algunos hogares admisibles no estuvieran seguros de serlo, muchos decidieron autoexcluirse de manera voluntaria, probablemente porque los pagos del programa no son suficientemente atractivos en relación con el costo de la vida en las zonas urbanas. Conviene recordar que el valor de los subsidios en efectivo es idéntico en las zonas urbanas y las rurales, a pesar de la evidente diferencia en el costo de la vida.

\section{Literatura aplicable}

Tal y como se comenta en la literatura, el índice de abandono de los PTC es mucho más elevado en zonas urbanas (con un índice anual de más del 7\%) que en zonas rurales (un índice anual del 3\%). Así pues, el índice de abandono total entre 2002 y 2007 se sitúa en torno al 43\% en zonas urbanas frente a solo el 16\% en entornos rurales (Álvarez, Devoto y Winters, 2008). González-Flores, Heracleous y Winters (2012) estiman que tres cuartas partes de las personas abandonan como consecuencia de sus propias decisiones, de donde un $56 \%$ lo hace porque no consiguieron cumplir los requisitos sanitarios y nutricionales y un $18 \%$ porque no consiguieron reunir otros requisitos de procedimiento. Por lo tanto, solo una cuarta parte de los abandonos del programa se pueden atribuir al proceso de recertificación de tres años (20\%) y a las auditorías (6\%) por parte de los administradores del programa para reducir los errores de inclusión.

De acuerdo con la teoría de la autofocalización, la relación entre la posición socioeconómica y la probabilidad de abandono del programa debería ser positiva y lineal. En efecto, las imposiciones asociadas al cumplimiento de las condiciones deberían actuar como mecanismo de autorregulación (González-Flores, Heracleous y Winters, 2012). Esto supone que los hogares menos necesitados deberían autoexcluirse del programa dado el costo de oportunidad relativamente elevado y la existencia de mejores alternativas (en caso de concurrir mejores aptitudes o mayores oportunidades migratorias, entre otros factores). Sin embargo, las conclusiones empíricas de González-Flores, Heracleous y Winters (2012) refutan la hipótesis de la autofocalización en el contexto de las zonas urbanas en México. A partir de datos de la administración del programa, estos autores describen una relación entre el puntaje (o índice de pobreza) y la probabilidad de abandono del programa que se expresa mediante una curva en forma de U. Además, los participantes más vulnerables (como las familias monoparentales) presentan la mayor probabilidad de abandono del programa. Estas últimas conclusiones sugieren que los costos directos e indirectos relacionados con el cumplimiento de los requisitos del programa, en combinación con una mala comprensión del proyecto y sus beneficios, han tenido un efecto muy negativo en la participación a largo plazo de los hogares más pobres. González-Flores, Heracleous y Winters (2016) destacan también que la mitad de los participantes urbanos decidió renunciar a las becas completas, ya que no consiguieron cumplir el requisito de enviar a sus hijos a la escuela. Este incumplimiento del componente educativo tiene una especial prevalencia entre los hogares más pobres y afecta especialmente las becas de educación secundaria. Esta conclusión pone de manifiesto que, para los adolescentes, el costo de oportunidad de estudiar en lugar de trabajar es mucho más elevado en las ciudades que en las zonas rurales. Según Schultz (2004), las becas proporcionadas en el marco del programa representan entre la mitad y un tercio de un sueldo a tiempo completo en las ciudades de México. 
González-Flores, Heracleous y Winters (2012) informan de una correlación positiva entre la probabilidad de abandono del programa y el tamaño de la ciudad. Argumentan que esto se puede deber en parte a la congestión característica de las grandes ciudades mexicanas, que se traduce en mayores costos de transporte directos (precio del desplazamiento) e indirectos (tiempo invertido). Además, puesto que en teoría el costo de vida aumenta en paralelo con el tamaño de la ciudad, es probable que el poder adquisitivo que ofrecen las transferencias monetarias (que son siempre iguales con independencia de la zona) disminuya al aumentar el tamaño de la ciudad. Dicho de otro modo, en grandes ciudades los incentivos en efectivo pueden no compensar los costos directos e indirectos derivados del cumplimiento de las condiciones impuestas. Por último, González-Flores, Heracleous y Winters (2012) observan que la probabilidad de abandono del programa tiene una correlación negativa con el nivel de marginación comunitaria (porcentaje de hogares pobres en la comunidad). Los autores sugieren que una posible explicación puede estar relacionada con la teoría de redes: puesto que la densidad de participantes es menor en comunidades menos marginales, es menos probable que las personas pobres en dichas comunidades obtengan información acerca del programa.

\section{Datos y estrategia empírica}

Los datos empleados en este estudio proceden de la Encuesta Nacional sobre Niveles de Vida de los Hogares (ENNVIH), la primera encuesta con una muestra representativa de la población mexicana a nivel nacional, rural, urbano y regional. Sus directrices de muestreo fueron elaboradas por el Instituto Nacional de Geografía y Estadística (INEGI). La encuesta contiene información correspondiente a un período de diez años, recopilada a través de tres levantamientos. El primer levantamiento de información fue implementado en el año 2002 y encuestó a 35.677 personas pertenecientes a 8.440 hogares ubicados en 150 localidades de 16 estados mexicanos. Dado el carácter longitudinal de la encuesta, en el segundo (2005/06) y el tercer (2009-2012) levantamiento se volvió a entrevistar a la muestra inicial de 2002, consiguiéndose ubicar y volver a entrevistar a casi un 90\% de los hogares de la muestra original. La encuesta incluye datos sobre la utilización del programa y el monto recibido anualmente por los participantes a través de transferencias monetarias, así como información detallada sobre las características socioeconómicas y demográficas de los hogares y sus componentes.

El principal objetivo de este estudio es evaluar de manera empírica la influencia de los incentivos en efectivo sobre la probabilidad de abandono del programa en zonas urbanas en México. En consecuencia, solo se examinaron aquellos hogares urbanos que estaban inscritos y recibieron transferencias monetarias en 2005. Aquellos que siguieron participando en el programa se diferenciaron posteriormente de los que lo abandonaron a través de información sobre el estado de tratamiento de la encuesta de 2012. Puesto que la ENNVIH es representativa a nivel rural y urbano, se asume que los hogares en la muestra urbana son representativos de los hogares participantes en todas las zonas urbanas de México. El modelo reducido basado en participantes urbanos adopta la forma de una regresión binomial probit:

$$
P\left(\text { Participante }_{j}\right)=\alpha+\beta_{1} X_{j}+\beta_{2} \text { Transferencias }_{j}+\varepsilon_{j}
$$

Donde $P\left(\right.$ Participante $_{j}$ ) es la probabilidad de que un hogar $j$ siga siendo participante entre 2005 y 2012 y no abandone el programa en dicho período. $X_{j}$ es un vector de variables de control medidas en 2005 que incluye las características socioeconómicas y demográficas del cabeza de familia (edad, género, renta anual del trabajo, estado civil, educación y categoría socioprofesional) y del hogar en su conjunto (número de hijos clasificados por grupo de edad, porcentaje de niñas, tasa de dependencia y activos en propiedad). El vector $X_{j}$ incluye también un factor comunitario para capturar el tamaño de las ciudades y su nivel de desarrollo urbano en 2005 (un índice compuesto que mide la disponibilidad 
de infraestructuras en la localidad). Por último, Transferencias $s_{j}$ es el valor total (en pesos mexicanos) de las transferencias que el hogar recibió en 2005. En teoría, los hogares que recibieron más efectivo fueron los que permanecieron más tiempo en el programa o los que acumularon más becas escolares (por tener hijos de mayor edad o niñas en edad escolar, véase el cuadro A1.1 en el anexo A1). Por este motivo, se incluyó un control para el número de hijos por grupo de edad y para el porcentaje de niñas en los hogares participantes. La variable de transferencias monetarias se analizó usando dos enfoques diferentes: lineal, por medio de una transformación logarítmica de los pagos anuales en efectivo, y no lineal, por medio de una categorización de los cuartiles de la distribución. Los valores extremos quedan excluidos de acuerdo con los límites razonables de transferencia basados en los valores estimados en el cuadro A1.1 en el anexo A1. Las variables utilizadas en el modelo se describen en el cuadro A1.2 en el anexo A1.

El objetivo de la ecuación (1) es comprobar las siguientes hipótesis:

- H1 (hipótesis de autofocalización): Para los hogares participantes, si la relación entre el estatus socioeconómico y la probabilidad de permanecer en el programa es negativa y lineal, esto quiere decir que los hogares menos necesitados se autoexcluyen del programa como consecuencia de un costo de oportunidad relativamente elevado y de la existencia de mejores alternativas. En este caso, se acepta la hipótesis de la autofocalización y la eficacia del programa se limita por errores de inclusión. Por otra parte, si la relación entre el estatus socioeconómico y la probabilidad de permanecer en el programa es positiva o se expresa mediante una curva en forma de $U$ invertida, la hipótesis de la autofocalización queda rechazada y la eficacia del programa se limita por errores de exclusión.

- H2 (hipótesis de falta de incentivos en efectivo): En caso de que las transferencias monetarias no sean suficientes para retener a los hogares más pobres dentro del programa (hogares que en teoría no se ven afectados por los procesos de autofocalización y recertificación), se podría asumir que los subsidios en efectivo no compensan las imposiciones asociadas al cumplimiento de las condiciones del programa. Para comprobar esta hipótesis se incluye un término de interacción en una especificación alternativa de la ecuación (1): el valor de las transferencias se cruza con el cuartil de ingresos del cabeza de familia.

En aras de la claridad y la transparencia, se debe discutir adecuadamente la solidez del modelo empírico en relación con los problemas de endogeneidad. Está claro que el intervalo de tiempo entre los regresores y el indicador de resultados descarta una potencial presencia de causalidad inversa en el modelo; no obstante, el hecho de que un participante permanezca o abandone en 2012 no afecta el valor de los pagos en efectivo efectuados en 2005 ni su estatus socioeconómico en 2005. Sin embargo, la omisión de factores que tienen una correlación simultánea con los regresores y la varianza no explicada de la probabilidad de permanecer en el programa $\left(\varepsilon_{j}\right)$ podría sesgar las estimaciones del modelo probit (ecuación (1)). Por ejemplo, se asume que la preocupación de los miembros del hogar por la educación y la salud y sus aptitudes cognitivas tienen una correlación positiva y simultánea con el estatus socioeconómico del hogar (incluido en $X_{j}$ ), con el valor acumulado de las becas (incluidas en los pagos en efectivo, Transferencias $s_{j}$ ) y con el grado de comprensión del programa e implicación en él (y, por tanto, con P(Participante $j$ )). De forma intuitiva, puede interpretarse que una omisión así podría causar una sobrevaloración de la relación positiva entre el estatus socioeconómico y la probabilidad de permanecer en el programa y, por lo tanto, resultar en el rechazo erróneo de la hipótesis de autofocalización (H1). En lo que respecta a H2, estos factores omitidos también podrían causar una sobrevaloración de la relación positiva entre las transferencias monetarias y la probabilidad de permanecer en el programa. Lamentablemente, estas intuiciones solo se pueden probar de manera parcial, puesto que se trata de factores que no se suelen observar. Sin embargo, el presente estudio trata de capturar estos sesgos indeseables mediante la inclusión de la máxima puntuación de aptitudes cognitivas obtenida por los miembros adultos del hogar en una especificación alternativa de 
la ecuación (1)¹. Además, como ya se mencionó, se tiene en cuenta el número de hijos por grupo de edad para limitar los sesgos de endogeneidad. La omisión de este factor podría haber resultado en una infravaloración del efecto positivo de los incentivos en efectivo sobre la probabilidad de permanecer en el programa (H2). En efecto, los hogares con hijos de más edad recibieron potencialmente una cantidad superior en concepto de becas en 2005 (véase el cuadro A1.1 del anexo 1) y, por lo tanto, presentan una probabilidad de abandono del programa más elevada durante el proceso de recertificación por la posibilidad de que sus hijos dejen de ser menores de edad (puesto que el puntaje se calcula a partir del número de hijos menores de edad).

Otra posible limitación de este estudio se refiere a la disminución de los datos del panel entre 2005 y 2012. No hay razón para asumir que muchos de los hogares que dejaron de participar en la encuesta (por haber migrado, por ejemplo) hubieran abandonado realmente el programa, de haberse incluido sus datos en la encuesta. Además, es probable que los potenciales abandonos del programa por parte de hogares que también dejaron la encuesta tengan las mismas características que los abandonos de los que permanecieron en ella. Por eso se considera que la disminución del panel no guarda correlación con la probabilidad de abandonar el programa y, por tanto, no afecta los resultados.

\section{Resultados}

\section{Estadística descriptiva}

Tal y como se esperaba, el índice de participación de los hogares en el programa (basado en las muestras totales) es notablemente mayor en zonas rurales que en comunidades urbanas (véase el cuadro 1); frente a una participación de aproximadamente una cuarta parte de los hogares rurales en el programa en el período 2002-2012, el índice de participación de los hogares urbanos creció del 3\% en 2002 al $6 \%$ en 2012. Este aumento está probablemente relacionado con la expansión gradual del programa. Aunque en las grandes ciudades (de más de 100.000 habitantes) el índice de participación se triplicó entre 2002 y 2012, en las ciudades medianas (entre 15.000 y 100.000 habitantes) este índice solo se duplicó y en las ciudades pequeñas (entre 2.500 y 15.000 habitantes) creció solo un 40\%. Además, el índice de participación se reduce a medida que aumenta el tamaño de la ciudad. De acuerdo con González-Flores, Heracleous y Winters (2012), el presente estudio concluye que la implementación del programa resultó difícil en los grandes centros urbanos.

\section{Cuadro 1}

México: índices de participación de los hogares en el programa de transferencias condicionadas, basados en muestras totales, 2002, 2005/06, 2009-2012

\begin{tabular}{|c|c|c|c|c|c|c|}
\hline & \multicolumn{2}{|r|}{2002} & \multicolumn{2}{|c|}{$2005 / 06$} & \multicolumn{2}{|c|}{$2009-2012$} \\
\hline & Núm. & $\begin{array}{c}\text { Tratados } \\
\text { (en porcentajes) }\end{array}$ & Núm. & $\begin{array}{c}\text { Tratados } \\
\text { (en porcentajes) }\end{array}$ & Núm. & $\begin{array}{c}\text { Tratados } \\
\text { (en porcentajes) }\end{array}$ \\
\hline Zonas urbanas & 10616 & 3 & 10598 & 4 & 10446 & 6 \\
\hline más de 100000 habitantes & 6812 & 1 & 6639 & 2 & 6475 & 3 \\
\hline entre 15000 y 100000 habitantes & 1863 & 3 & 1849 & 5 & 1866 & 6 \\
\hline entre 2500 y 15000 habitantes & 1941 & 9 & 2110 & 10 & 2105 & 13 \\
\hline Zonas rurales & 7834 & 25 & 7969 & 26 & 8304 & 26 \\
\hline \multirow{2}{*}{$\begin{array}{l}\text { Test de comparación de medias rural y urbana } \\
\text { (valor p) }\end{array}$} & & 22 & & 22 & & 20 \\
\hline & & $(0,000)$ & & $(0,000)$ & & $(0,000)$ \\
\hline
\end{tabular}

Fuente: Elaboración propia, sobre la base de Universidad Iberoamericana (IBERO)/Centro de Investigación y Docencia Económicas (CIDE), "Encuesta Nacional sobre Niveles de Vida de los Hogares (ENNVIH)" [en línea] http://www.ennvih-mxfls.org.

\footnotetext{
1 Las puntuaciones de destrezas cognitivas no se incluyen en el modelo principal por el bajo número de observaciones para esta variable.
} 
El cuadro 2 muestra que los índices de abandono del programa son notablemente más altos en las ciudades que en las zonas rurales. Se podría concluir que esta gran diferencia se debe a que la pobreza es menos prevalente en las zonas urbanas que en las zonas rurales. No obstante, diversos elementos sugieren que también se podría explicar porque los incentivos en efectivo en los entornos urbanos no compensan las imposiciones asociadas con el cumplimiento de las condiciones requeridas. En efecto, el costo de la vida difiere notablemente entre las zonas rurales y las urbanas (véase el cuadro A1.3 en el anexo A1).

\section{Cuadro 2}

México: índices de abandono del programa de transferencias condicionadas entre los hogares, 2005/06 y 2009-2012

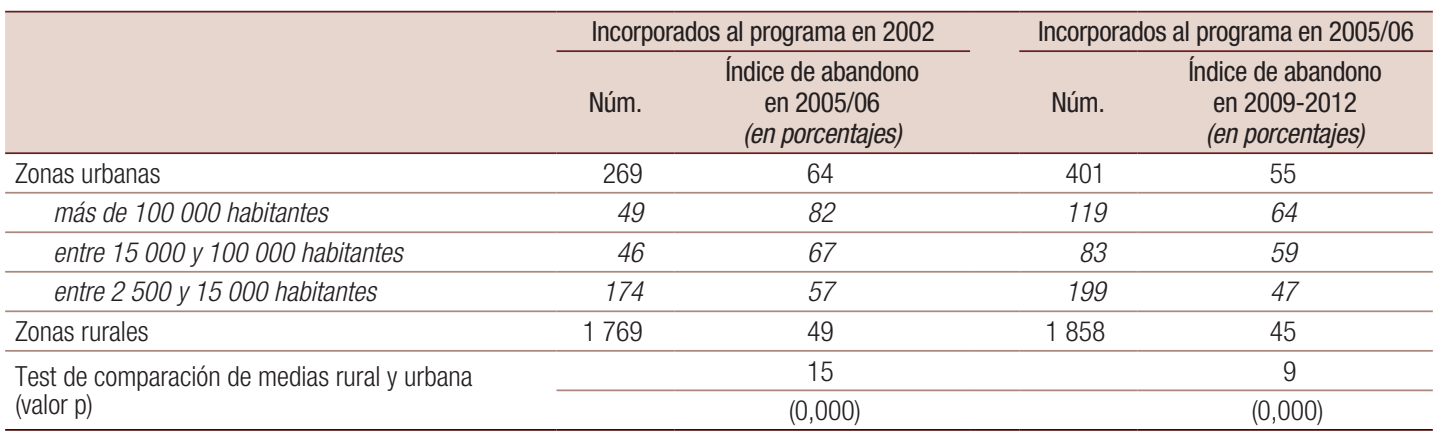

Fuente: Elaboración propia, sobre la base de Universidad Iberoamericana (IBERO)/Centro de Investigación y Docencia Económicas (CIDE), "Encuesta Nacional sobre Niveles de Vida de los Hogares (ENNVIH)" [en línea] http://www.ennvihmxfls.org.

A pesar de esto, el cuadro 3 no muestra diferencias significativas entre los pagos en las zonas rurales y las urbanas en 2005. Además, la distribución de las transferencias monetarias es similar en las dos zonas (véase el gráfico A1.1 en el anexo A1) y el estatus socioeconómico del cabeza de familia no afecta de manera significativa al valor de los incentivos en efectivo (véase el cuadro A1.4 en el anexo A1). La falta de correlación entre estatus socioeconómico y transferencias monetarias indica que los importes de los pagos no son proporcionales al nivel de pobreza, lo cual significa que cada persona beneficiaria tiene derecho al mismo monto. También sugiere que no existe una corrupción significativa en el seno del programa: no se percibe un grupo de participantes más ricos que reciban una porción desproporcionadamente grande de los pagos en efectivo.

\section{Cuadro 3}

México: transferencias monetarias anuales por hogar del programa de transferencias condicionadas, 2005/06

(En pesos mexicanos)

\begin{tabular}{lccccc}
\hline & Núm. & Media & Cuartil I & Mediana & Cuartil III \\
\hline Zonas urbanas & 155 & 3299 & 1500 & 2040 & 4800 \\
\hline Zonas rurales & 662 & 3286 & 1800 & 2040 & 4200 \\
\hline $\begin{array}{l}\text { Test de comparación de medias rural vs. urbana } \\
\text { (valor p) }\end{array}$ & \multicolumn{5}{c}{12,23} \\
\hline
\end{tabular}

Fuente: Elaboración propia, sobre la base de Universidad Iberoamericana (IBERO)/Centro de Investigación y Docencia Económicas (CIDE), "Encuesta Nacional sobre Niveles de Vida de los Hogares (ENNVIH)" [en línea] http://www.ennvihmxfls.org. 


\section{Determinantes de los abandonos del programa}

Puesto que el principal objetivo de este estudio consiste en evaluar el impacto de los pagos en efectivo sobre la probabilidad de que los participantes permanezcan en el programa de transferencias condicionadas de México o lo abandonen, solo se analizan aquellos hogares que estaban participando en el programa durante la encuesta de 2005/06. Como siguiente paso, se identificó a los participantes que permanecieron en el programa y a los que lo abandonaron usando información sobre el estado de tratamiento de la encuesta de 2012. Se empleó una regresión probit para estimar la relación entre las transferencias monetarias anuales recibidas un año antes de la encuesta de 2005/06 y la probabilidad de permanecer en el programa en lugar de abandonarlo entre 2005/06 y 2009-2012 (véase el cuadro 4). Se aplicó un control para la presencia de heterogeneidad entre los participantes que permanecieron y los que lo abandonaron mediante el uso de un conjunto integral de variables recopiladas durante la encuesta de 2005/06. Como ya se mencionó anteriormente, estas variables de control abordan parcialmente los supuestos problemas de endogeneidad. Estas covariantes incluyen características en lo que respecta al cabeza de familia, al hogar y al municipio. Los errores estándar se agrupan en el nivel del municipio para compensar una potencial correlación intracomunitaria, como por ejemplo instalaciones de inscripción específicas. El número de observaciones es relativamente pequeño debido a la baja tasa de participación en las ciudades mexicanas. Por lo tanto, la muestra urbana cuenta con 98 hogares inscritos. No obstante, las estimaciones del modelo probit son de la calidad adecuada: los pseudo- $\mathrm{R}^{2}$ son elevados (entre el $45 \%$ y el $58 \%)^{2}$. Pese al bajo número de observaciones, los errores estándar no son muy elevados y diversas variables explicativas son significativas. Este último punto no llama la atención, ya que todos los hogares participantes presentan características relativamente homogéneas: tanto los participantes que permanecieron en el programa como los que lo abandonaron son hogares pobres.

\section{Cuadro 4}

México: determinantes de la probabilidad de permanecer en el programa de transferencias condicionadas en comparación con los de la probabilidad de abandonarlo, zonas urbanas

(Efectos marginales medios)

\begin{tabular}{|c|c|c|c|c|c|}
\hline \multirow{2}{*}{ Variable dependiente } & \multicolumn{5}{|c|}{ Permanecer en el programa frente a abandonarlo, entre 2005 y 2012} \\
\hline & (1) & (2) & (3) & (4) & (5) \\
\hline \multirow[t]{2}{*}{ Edad del cabeza de familia (CF) } & $0,0385^{\star \star \star}$ & $0,0352^{* \star *}$ & $0,111^{\star * *}$ & $0,0430^{* \star *}$ & $0,0468^{\star \star \star}$ \\
\hline & $(2,980)$ & $(3,011)$ & $(3,481)$ & $(5,416)$ & $(4,520)$ \\
\hline \multirow[t]{2}{*}{ Sexo del CF (hombre) } & $-0,692^{\star \star \star}$ & $-0,713^{\star \star \star}$ & $-0,955^{\star \star \star}$ & $-0,515^{*}$ & $-0,689^{\star \star \star}$ \\
\hline & $(-3,396)$ & $(-3,725)$ & $(-3,333)$ & $(-1,804)$ & $(-4,078)$ \\
\hline \multirow[t]{2}{*}{ Estado civil del CF (en pareja) } & $0,625^{\star \star \star}$ & $0,597^{\star \star \star}$ & $0,797^{\star \star *}$ & $0,582^{\star \star \star}$ & $0,621^{\star * *}$ \\
\hline & $(3,880)$ & $(4,096)$ & $(3,246)$ & $(3,415)$ & $(3,945)$ \\
\hline \multirow[t]{2}{*}{ Número de niños entre 0 y 5 años } & $0,161^{*}$ & $0,171^{*}$ & $0,469^{\star \star \star}$ & 0,0486 & $0,146^{*}$ \\
\hline & $(1,872)$ & $(1,946)$ & $(2,834)$ & $(0,527)$ & $(1,768)$ \\
\hline \multirow[t]{2}{*}{ Número de niños entre 6 y 12 años } & $0,260^{\star \star \star}$ & $0,260^{\star *}$ & $0,501^{\star \star \star}$ & $0,226^{\star \star}$ & $0,372^{\star \star \star}$ \\
\hline & $(2,718)$ & $(2,490)$ & $(3,529)$ & $(2,406)$ & $(2,913)$ \\
\hline \multirow[t]{2}{*}{ Número de adolescentes entre 13 y 15 años } & $-0,0152$ & 0,0150 & 0,0357 & $-0,0365$ & $-0,0684$ \\
\hline & $(-0,146)$ & $(0,132)$ & $(0,253)$ & $(-0,376)$ & $(-0,522)$ \\
\hline \multirow[t]{2}{*}{ Porcentaje de niñas (de 0 a 15 años) } & $-0,00126$ & $-0,00117$ & $-0,000330$ & 0,000760 & $-0,00119$ \\
\hline & $(-0,629)$ & $(-0,553)$ & $(-0,116)$ & $(0,348)$ & $(-0,494)$ \\
\hline \multirow[t]{2}{*}{ Tasa edad-dependencia } & $-0,138$ & $-0,140$ & $-0,791^{\star \star \star}$ & $-0,0168$ & $-0,0573$ \\
\hline & $(-1,461)$ & $(-1,099)$ & $(-3,005)$ & $(-0,137)$ & $(-0,465)$ \\
\hline
\end{tabular}

\footnotetext{
2 Al ejecutar regresiones lineales de la ecuación (1) (con base en un estimador por mínimos cuadrados), observamos que los $\mathrm{R}^{2}$ presentan valores similares a los pseudo- $\mathrm{R}^{2}$ (no mostrados). Esto quiere decir que la varianza de los regresores seleccionados explica en torno al 45\%-60\% de la varianza en la probabilidad de permanecer en el programa. Los $R^{2}$ y pseudo- $R^{2}$ más elevados se encuentran en la especificación que incluye las puntuaciones de aptitudes cognitivas más elevadas obtenidas por miembros adultos de un hogar (véase el cuadro 4, columna 3).
} 
Cuadro 4 (conclusión)

\begin{tabular}{|c|c|c|c|c|c|}
\hline \multirow{2}{*}{ Variable dependiente } & \multicolumn{5}{|c|}{ Permanecer en el programa frente a abandonarlo, entre 2005 y 2012} \\
\hline & (1) & (2) & (3) & (4) & (5) \\
\hline \multirow[t]{2}{*}{ CF carece de educación } & 0,132 & 0,125 & $-0,180$ & 0,0340 & 0,275 \\
\hline & $(0,787)$ & $(0,809)$ & $(-0,768)$ & $(0,216)$ & $(1,507)$ \\
\hline \multirow{2}{*}{$\begin{array}{l}\text { Máxima puntuación de aptitudes cognitivas obtenida } \\
\text { por los miembros adultos del hogar }\end{array}$} & \multicolumn{5}{|c|}{$-0,00891^{*}$} \\
\hline & \multicolumn{5}{|c|}{$(-1,742)$} \\
\hline \multirow[t]{2}{*}{ Trabajador informal asalariado (CF) ${ }^{\mathrm{a}}$} & $0,401^{\star *}$ & $0,398^{\star *}$ & $0,592^{\star *}$ & $0,388^{*}$ & $0,654^{\star \star \star}$ \\
\hline & $(2,512)$ & $(2,448)$ & $(2,391)$ & $(1,713)$ & $(3,290)$ \\
\hline \multirow[t]{2}{*}{ Trabajador formal asalariado $(\mathrm{CF})^{\mathrm{a}}$} & $0,472^{*}$ & $0,507^{*}$ & $0,749^{\star \star *}$ & $0,565^{\star \star}$ & $0,589^{* *}$ \\
\hline & $(1,777)$ & $(1,838)$ & $(3,329)$ & $(2,032)$ & $(2,324)$ \\
\hline \multirow[t]{2}{*}{ Trabajador por cuenta propia (CF) ${ }^{\mathrm{a}}$} & $0,503^{\star \star \star}$ & $0,545^{\star \star \star}$ & $0,763^{\star \star \star}$ & $0,577^{\star \star \star}$ & $0,756^{\star \star \star}$ \\
\hline & $(3,940)$ & $(4,078)$ & $(3,199)$ & $(3,080)$ & $(4,303)$ \\
\hline \multirow[t]{2}{*}{ Renta del trabajo del CF-cuartil I } & & & & & $1,000^{\text {***}}$ \\
\hline & & & & & $(3,247)$ \\
\hline \multirow[t]{2}{*}{ Renta del trabajo del CF-cuartil II } & $0,691^{\star \star}$ & $0,673^{\star \star}$ & $0,980^{\star \star \star}$ & $-1,000^{\star \star \star}$ & \\
\hline & $(2,482)$ & $(2,117)$ & $(3,192)$ & $(-2,582)$ & \\
\hline \multirow[t]{2}{*}{ Renta del trabajo del CF-cuartil III } & 0,457 & 0,394 & $0,902^{\star \star \star}$ & $-0,989^{\star \star}$ & \\
\hline & $(1,633)$ & $(1,274)$ & $(2,814)$ & $(-2,534)$ & \\
\hline \multirow[t]{2}{*}{ Renta del trabajo del CF-cuartil IV } & $0,521^{*}$ & 0,465 & $0,948^{\star \star \star}$ & $-0,997^{\star \star}$ & \\
\hline & $(1,708)$ & $(1,362)$ & $(3,094)$ & $(-2,252)$ & \\
\hline \multirow[t]{2}{*}{ Índice de activos en propiedad } & $-1,017^{\star \star \star}$ & $-1,020^{\star \star \star}$ & $-1,319^{\star \star *}$ & $-0,859^{\star \star \star}$ & $-1,501^{\star * \star}$ \\
\hline & $(-3,229)$ & $(-3,112)$ & $(-3,406)$ & $(-3,478)$ & $(-3,456)$ \\
\hline \multirow[t]{2}{*}{ Índice de activos en propiedad al cuadrado } & $0,159^{\star \star \star}$ & $0,157^{\star \star \star}$ & $0,197^{\star \star \star}$ & $0,141^{\star \star \star}$ & $0,222^{\star \star \star}$ \\
\hline & $(3,689)$ & $(3,554)$ & $(3,478)$ & $(3,799)$ & $(3,475)$ \\
\hline \multirow[t]{2}{*}{ Índice de desarrollo de infraestructuras } & $-0,210^{\star \star \star}$ & $-0,221^{\star \star \star}$ & $-0,295^{\star \star}$ & $-0,164^{\star \star}$ & $-0,188^{* *}$ \\
\hline & $(-3,374)$ & $(-3,665)$ & $(-2,303)$ & $(-2,418)$ & $(-2,144)$ \\
\hline \multirow[t]{2}{*}{$\log$ (transferencias) } & $0,228^{\star \star \star}$ & & & $-0,479^{\star \star \star}$ & $0,513^{\star \star \star}$ \\
\hline & $(3,049)$ & & & $(-2,711)$ & $(3,276)$ \\
\hline \multirow[t]{2}{*}{ Transferencias cuartil II (1 500-2 040 pesos/año) } & & 0,247 & $0,722^{\star \star}$ & & \\
\hline & & $(1,157)$ & $(2,136)$ & & \\
\hline \multirow[t]{2}{*}{ Transferencias cuartil III (2 040-4 800 pesos/año) } & & 0,127 & 0,256 & & \\
\hline & & $(0,633)$ & $(1,097)$ & & \\
\hline \multirow[t]{2}{*}{ Transferencias cuartil IV (>4 800 pesos/año) } & & $0,548^{\star \star \star}$ & $0,799^{\star \star \star}$ & & \\
\hline & & $(3,905)$ & $(4,955)$ & & \\
\hline \multirow[t]{2}{*}{$\log \left(\right.$ transferencias) ${ }^{\star}$ Cuartil de ingresos I } & & & & & $-1,107^{\star \star \star}$ \\
\hline & & & & & $(-3,660)$ \\
\hline \multirow[t]{2}{*}{$\log \left(\right.$ transferencias) ${ }^{\star}$ Cuartil de ingresos II } & & & & $0,856^{\star \star \star}$ & \\
\hline & & & & $(2,937)$ & \\
\hline \multirow[t]{2}{*}{$\log \left(\right.$ transferencias) ${ }^{\star}$ Cuartil de ingresos III } & & & & $0,748^{\star \star \star}$ & \\
\hline & & & & $(2,906)$ & \\
\hline \multirow[t]{2}{*}{$\log \left(\right.$ transferencias) ${ }^{*}$ Cuartil de ingresos III } & & & & $0,639^{\star \star}$ & \\
\hline & & & & $(2,519)$ & \\
\hline Observaciones & 98 & 98 & 89 & 98 & 98 \\
\hline Pseudo-R² & 44,84 & 46,91 & 58,37 & 47,70 & 54,42 \\
\hline
\end{tabular}

Fuente: Elaboración propia, sobre la base de Universidad Iberoamericana (IBERO)/Centro de Investigación y Docencia Económicas (CIDE), "Encuesta Nacional sobre Niveles de Vida de los Hogares (ENNVIH)" [en línea] http://www.ennvihmxfls.org.

Nota: Las estadísticas z robustas y por conglomerados (a nivel de municipio) se presentan entre paréntesis: ${ }^{* *} p<0,01$, ${ }^{* *} p<0,05,{ }^{*} p<0,1$.

a El grupo de referencia incluye cabezas de familia inactivos, desempleados y no retribuidos.

Las columnas 1 y 2 del cuadro 4 presentan los resultados de la ecuación (1). Los efectos marginales de las variables de control son coherentes con los resultados de González-Flores, Heracleous y Winters (2012). Por ejemplo, cuanto mayor sea el desarrollo en infraestructura de una localidad urbana, más alta tenderá a ser la probabilidad. Es probable que la concentración de participantes en 
las comunidades más marginadas se traduzca en una mejor difusión y comprensión del programa. Además, como se esperaba, la presencia de varios niños pequeños y preadolescentes en el hogar incrementa notablemente la probabilidad de que el hogar permanezca en el programa entre 2005/06 y 2009-2012. Resulta interesante que el porcentaje de niñas no influya en la probabilidad de permanecer en el programa. Habría cabido esperar que los hogares con varias niñas que reciben becas escolares estuvieran más interesados en permanecer, puesto que las becas para niñas son más elevadas que las becas para niños (véase el cuadro A1.1 en el anexo A1). En lo que respecta a las características de los cabezas de familia, nuestros resultados vienen a apoyar las conclusiones de González-Flores, Heracleous y Winters (2012): cuando la cabeza de familia es una mujer, tiene pareja y es mayor, la probabilidad de permanecer en el programa aumenta notablemente. Frente a esto, los cabezas de familia que pertenecen a categorías laborales más bajas (personas inactivas, desempleadas o no remuneradas) presentan un mayor riesgo de abandono del programa. En relación con los ingresos del trabajo del cabeza de familia, el $75 \%$ más rico tiene más probabilidades de permanecer en el programa que el $25 \%$ más pobre. La probabilidad de permanecer es especialmente elevada para el cuartil II en comparación con el cuartil I (de 67 a 69 puntos porcentuales más alta). Esta ausencia de linealidad se discute ampliamente en González-Flores, Heracleous y Winters (2012), quienes observan una relación entre el puntaje y el riesgo de abandono del programa en zonas urbanas que se expresa mediante una curva en forma de U. En resumen, en línea con el importante trabajo de González-Flores, Heracleous y Winters (2012), nuestros resultados ponen de manifiesto las dificultades de dar a conocer el programa entre los participantes más vulnerables (personas inactivas, desempleadas y pobres) y de fomentar su adhesión al programa y su permanencia en él ${ }^{3}$. Dado que los participantes más pobres muestran una probabilidad de abandono del programa especialmente elevada, se puede rechazar la hipótesis de la autofocalización ( $\mathrm{H} 1$ rechazada).

En la columna 1 del cuadro 4, la variable transferencias monetarias anuales se expresa en forma lineal y logarítmica. Encontramos que el valor de los pagos aumenta la probabilidad de permanecer en el programa (significativo al 1\%). Una unidad adicional del logaritmo de transferencias aumenta la probabilidad de permanecer en el programa 23 puntos porcentuales. En la columna 2 del cuadro 4, cuando el valor de las transferencias se expresa en categorías de cuartiles, observamos que los hogares del cuartil IV, que recibieron más de 4.800 pesos mexicanos en 2005, tienen una mayor probabilidad de permanecer en el programa que los hogares del cuartil I, que recibieron menos de 1.500 pesos mexicanos. La brecha entre los cuartiles I y IV es de aproximadamente 55 puntos porcentuales (significativo al 1\%). Sin embargo, no existe una diferencia significativa entre los primeros tres cuartiles de transferencias monetarias (cuartiles I, II y III).

La columna 3 del cuadro 4 muestra una especificación alternativa del modelo que tiene en cuenta la máxima puntuación de aptitudes cognitivas obtenida por los miembros adultos del hogar. Contrariamente a lo esperado (véase la sección IV) este factor reduce la probabilidad de permanecer en el programa, si bien el coeficiente ajustado solo es significativo al 10\%. Cabría especular que los hogares formados por adultos con altas aptitudes cognitivas tienen más probabilidades de abandonar el programa porque pueden evaluar mejor el desequilibrio entre los pagos monetarios y los costos derivados de las condiciones. Además, esta dotación de aptitudes cognitivas aumenta potencialmente la probabilidad de encontrar oportunidades más atractivas desde un punto de vista económico que las transferencias monetarias sociales (por ejemplo, oportunidades de empleo), algo que es en cierta medida coherente con la teoría de la autofocalización (véase la sección II.2). Así que, ligeramente en

\footnotetext{
3 No obstante, el cuadro 4 muestra que los hogares con menos activos y los hogares con más activos tienen más probabilidades de permanecer que aquellos con una cantidad intermedia de activos. Esta relación entre los activos en propiedad y la probabilidad de permanencia, que se expresa mediante una curva en forma de $U$, no es coherente con nuestros supuestos. Sin embargo, por lo que respecta a la proporción, muy pocos hogares se posicionan en el lado izquierdo de la distribución de activos: solo un $7 \%$ de los hogares poseen entre 0 y 2 activos (resultados disponibles previa solicitud). Por lo tanto, esta relación expresada mediante una curva en forma de U podría estar provocada por la presencia de casos extremos.
} 
contra de lo que cabría imaginar, si se omiten las aptitudes cognitivas de la ecuación (resultados de las columnas 1 y 2) se infravaloran los efectos positivos de los pagos en efectivo y de la renta del trabajo del cabeza de familia sobre la probabilidad de permanecer en el programa. En efecto, se presume que las aptitudes cognitivas mantienen una correlación positiva con los pagos en efectivo y los ingresos, pero una correlación negativa con la participación en el programa. Tal y como se explica en la sección IV, algunos factores adicionales como el nivel de preocupación por la salud y la educación podrían seguir sin observarse, lo cual se traduciría en un sesgo de los resultados que sobrevaloraría los efectos positivos de los ingresos del cabeza de familia sobre la probabilidad de permanecer en el programa. Sin embargo, dada la gran significación y magnitud de los coeficientes estimados en el cuadro 4, se asume que tales factores omitidos no sesgan las estimaciones de manera significativa.

Por último, las columnas 4 y 5 del cuadro 4 presentan unas especificaciones alternativas que introducen términos de interacción entre la renta del trabajo del cabeza de familia y el nivel del logaritmo de transferencias. A priori, la renta del trabajo del cabeza de familia afecta de manera significativa la relación entre los incentivos en efectivo y la probabilidad de permanecer en el programa. Más concretamente, la columna 4 muestra que las transferencias monetarias incrementan de manera significativa la probabilidad de permanecer en el programa para aquellos hogares que se sitúan en los tres cuartiles superiores de ingresos (cuartiles II, III y IV) en comparación con el más pobre (cuartil I). Del mismo modo, la columna 5 indica que un incremento de una unidad en el logaritmo de transferencias hace disminuir 0,6 puntos porcentuales la probabilidad de que el 25\% más pobre permanezca, en comparación con el $75 \%$ más rico (el coeficiente es significativo al 1\%) ${ }^{4}$. Estos resultados constituyen nuestra contribución más destacada a la literatura existente. En resumen, los hogares más pobres son especialmente sensibles a los bajos montos de pagos que caracterizan a la versión urbana del programa. Suponemos que las transferencias monetarias urbanas no consiguen compensar las imposiciones que implica el cumplimiento de las condiciones requeridas (como costos directos e indirectos o la inversión de tiempo) y la preferencia por gratificaciones inmediatas, lo cual se traduce en el abandono del programa por parte de los hogares más pobres ( $\mathrm{H} 2$ aceptada).

\section{Conclusiones}

En línea con el importante trabajo de González-Flores, Heracleous y Winters (2012), encontramos una relación positiva entre los ingresos del cabeza de familia y la probabilidad de permanecer en el programa para los hogares mexicanos de zonas urbanas. Esta tendencia positiva refuta la teoría de la autofocalización e indica la existencia de importantes errores de exclusión. Esta conclusión resulta fundamental para los administradores del programa, ya que cuestiona la eficacia del programa en su formato actual: a pesar de sus objetivos, el programa no consigue llegar a los hogares más pobres residentes en zonas urbanas y retenerlos.

El presente estudio contribuye a la literatura existente al llamar la atención, por medio de un análisis empírico, sobre el papel de los incentivos en efectivo como determinantes clave del preocupante alto número de abandonos del programa en zonas urbanas. En línea con la teoría sobre la utilización, nuestros resultados ponen de relieve un desequilibrio entre los incentivos en efectivo y las imposiciones asociadas a las condiciones requeridas que incrementa notablemente los errores de exclusión y los casos de abandono del programa en las zonas urbanas de México. Por debajo de un determinado umbral es posible que los subsidios en efectivo no compensen las imposiciones asociadas al cumplimiento de las condiciones (tales como costos directos e indirectos) y, por tanto, pueden incrementar el riesgo de abandonar el programa. Además, los bajos incentivos en efectivo que se ofrecen a los hogares

$4 \frac{\Delta \text { Prob(permanecer })}{\Delta \log (\text { transferencias })}=0,5113+(-1,107) * \Delta$ IngresosQ1 $=0,5113+(-1,107) * 1=-0,5957$. 
en las ciudades mexicanas afectan especialmente la capacidad de permanecer en el programa en el caso de los más pobres. Esto indica que el cumplimiento de las condiciones resulta especialmente difícil para los hogares más pobres y desfavorecidos (como suponen González-Flores, Heracleous y Winters (2012)). Además de la aparente incapacidad del programa para retener a los participantes más pobres, los insuficientes incentivos en efectivo pueden incluso disuadir a muchos hogares pobres de llegar a adherirse. Así pues, podemos concluir que el valor relativamente bajo de las transferencias monetarias en zonas urbanas explica el bajo índice de participación y el elevado índice de abandono del programa.

Los resultados sugieren que en las ciudades mexicanas los incentivos en efectivo son demasiado bajos (los montos son los mismos que se abonan a los hogares rurales) como para compensar las diferentes imposiciones asociadas al cumplimiento de las condiciones requeridas. Dado que el costo de la vida es más elevado en las ciudades que en las zonas rurales de México, recomendamos adaptar el valor de las transferencias monetarias al costo de la vida urbana. Aumentar el valor de las transferencias en las zonas urbanas puede no solo fomentar que se adhieran más hogares que reúnen las condiciones para participar, sino también reducir el número de abandonos del programa entre los beneficiarios más pobres. Además, reducir el número de abandonos del programa y de errores de exclusión es de interés público por motivos que van más allá de la lucha contra la pobreza. Por ejemplo, un estudio reciente muestra que la participación a largo plazo en el programa tiene un efecto protector contra la ganancia de peso (siendo que la obesidad es un enorme problema en países emergentes como México), mientras que la participación a corto plazo incrementa el riesgo de ganancia de peso (Levasseur, 2019). Resulta evidente que es necesario realizar análisis adicionales para determinar el valor adecuado de las transferencias monetarias para los hogares urbanos. Con objeto de minimizar las externalidades negativas que podría generar un pago adicional en efectivo, tales como flujos migratorios o errores de inclusión, los administradores del programa deberían adoptar diversas precauciones. Por ejemplo, el valor de los pagos en efectivo habría de ser proporcional al costo de la vida en el municipio y al nivel de pobreza del hogar admisible. Además de un potencial efecto desincentivador de la migración, estos pagos proporcionales podrían reducir el riesgo de abandono del programa en el caso de los hogares más pobres que viven en las zonas más caras. Por último, una conclusión importante que se debería tener en cuenta es que el riesgo de abandono del programa aumenta en forma paralela a la vulnerabilidad del hogar y el tamaño de la ciudad.

\section{Bibliografía}

Álvarez, C., F. Devoto y P. Winters (2008), "Why do beneficiaries leave the safety net in Mexico? A study of the effects of conditionality on dropouts", World Development, vol. 36, № 4, Ámsterdam, Elsevier.

Angelucci, M. y O. Attanasio (2009), "Oportunidades: program effect on consumption, low participation, and methodological issues", Economic Development and Cultural Change, vol. 57, № 3, Chicago, The University of Chicago Press.

Angelucci, M., O. Attanasio y V. Di Maro (2012), "The impact of Oportunidades on consumption, savings and transfers", Fiscal Studies, vol. 33, No 3, Oxford, Blackwell Publishing.

Attanasio, O., C. Meghir y N. Schady (2010), "Mexico's conditional cash transfer programme", The Lancet, vol. 375, No 9719, Ámsterdam, Elsevier, 20 de marzo.

Banerjee A. y E. Duflo (2011), Poor Economics: A Radical Rethinking of the Way to Fight Global Poverty, Nueva York, Public Affairs.

Behrman, J. R. y otros (2012), "Are conditional cash transfers effective in urban areas? Evidence from Mexico", Education Economics, vol. 20, №3, Londres, Routledge, Taylor \& Francis.

Fiszbein, A. y otros (2009), "Conditional cash transfers: reducing present and future poverty", A World Bank Policy Research Report, 47603, Washington, D.C., Banco Mundial. 
González-Flores, M., M. Heracleous y P. Winters (2016), "Conditional cash transfers and schooling decisions: evidence from urban Mexico", IDB Working Paper Series, № 721, Washington, D.C., Banco Interamericano de Desarrollo (BID).

- (2012), "Leaving the safety net: an analysis of dropouts in an urban conditional cash transfer program", World Development, vol. 40, № 12, Ámsterdam, Elsevier.

Hernández, D., J. Gómez de León y G. Vásquez (1999), Más oportunidades para las familias pobres: evaluación de resultados del Programa de Educación, Salud y Alimentación: primeros avances, Ciudad de México, Secretaría de Desarrollo Social.

Hoddinott, J. y L. Bassett (2008), "Conditional cash transfer programs and nutrition in Latin America: assessment of impacts and strategies for improvement", SSRN Scholarly Paper, № 1305326, Rochester, Social Science Research Network [en línea] https://papers.ssrn.com/sol3/papers.cfm?abstract_id=1305326.

Levasseur, P. (2019), "Can social programs break the vicious cycle between poverty and obesity? Evidence from urban Mexico", World Development, vol. 113, Ámsterdam, Elsevier.

Orozco, M. y C. Hubert (2005), "La focalización en el Programa de Desarrollo Humano Oportunidades de México", Serie de Documentos de Discusión sobre la Protección Social, 32761, № 0531, Washington, D.C., Banco Mundial.

Schultz, T. P. (2004), "School subsidies for the poor: evaluating the Mexican Progresa poverty program", Journal of Development Economics, vol. 74, № 1, Ámsterdam, Elsevier.

Skoufias, E. (2005), "PROGRESA and its impacts on the welfare of rural households in Mexico", Research Report, 139, Washington, D.C., Instituto Internacional de Investigación sobre Políticas Alimentarias (IFPRI).

Skoufias, E., B. Davis y S. de la Vega (2001), "Targeting the poor in Mexico: an evaluation of the selection of households into PROGRESA", World Development, vol. 29, № 10, Ámsterdam, Elsevier. 